\title{
Advances in Biomedical Imaging and Image Fusion
}

\author{
Leena Chandrashekar \\ Research Scholar, RVCE \\ Bangalore, India
}

\author{
A. Sreedevi \\ Associate Professor, EEE Dept, RVCE \\ Bangalore, India
}

\begin{abstract}
Biomedical imaging is a series of procedures which create images of the human body, or parts of the body, to help screen for possible illness or injury, diagnose the likely cause of symptoms and monitor health conditions or the effects of treatment. The objective of the paper is to provide an overview about various bio medical imaging techniques used in detection and diagnosis of Cancer. Each of these imaging techniques provides information about the anatomy, chemical or physiologic phenomena of the human body which are studied independently by doctors to identify Cancer. The biomedical imaging systems, applications, benefits, drawbacks and research challenges are discussed. Image Fusion and its role in Bio medical imaging is also discussed. Image Fusion is the process of fusing two or more bio medical images which contain complementary information into a single composite image. These enrich image quality and avoid redundancy thereby increase the clinical applicability of medical images for cancer detection, prognosis and treatment planning of Cancer.
\end{abstract}

\section{Keywords}

CT, MRI, PET, SPECT, Ultrasound imaging, Biomedical Image Fusion

\section{INTRODUCTION}

Around 1.7 crore people suffer from cancer worldwide. India has approximately 20-25 lakhs cancer cases any given time and nearly half of them die every year. Around 7 lakh new cancer cases are diagnosed every year in India. The number of deaths per year is projected to shoot up to 1.2 million by 2035 [1]. The most common, nearly half of all are cancers of the lung and oral cavity in men, and of the breast and cervix in women. The rates of occurrences of breast and cervical cancers are 25.8 and 22. Almost three of five cancer deaths in India are associated with tobacco or infectious diseases. Tobacco use alone accounts for about 40 per cent of all cancers in India. Around 95 per cent of the medical colleges in India do not have comprehensive cancer care services, comprising Surgical, Medical and Radiation Oncology departments, in the same campus. Currently there are around 2,000 medical and radiation oncologists in India - one per 5000 newly diagnosed cancer patients - and in almost all remote or rural areas even the most basic cancer treatment facilities are non-existent. As a result, urban cancer centres are overcrowded and underresourced, leading to long waiting times, delayed diagnoses, and treatment that comes too late for many patients.

Cancer is an abnormal growth of cells. In spite of space constraint, Cancer cells rapidly replicate and continue to utilize the nutrients shared by other cells. In addition to spreading in many areas of the body, Cancer cells do not function normally and look dissimilar from the normal cells. Oncology is the study of cancer. The tumors are classified into malignant and benign. The malignant tumor reproduces at a fast rate and can cause harm to the body, including death. Benign tumors grows slowly and do not spread to other parts of the body. The malignant tumors may be locally invasive or metastatic. The locally invasive tumor can attack the tissues nearby it, whereas, the metastatic tumor can send cells into other tissues in the body which are distant from the original tumor or primary tumor. The blood (circulatory system) or lymphatic system transports the cancerous cells to other parts of the body to form secondary tumors. Studies indicate Liver is the first organ to get affected earliest with secondary tumors.

Based on their origin, cancer can be classified broadly into 5 categories. Carcinomas are cancerous cells that have an effect on the tissues, organs and glands which account for $80 \%-90 \%$ of all cancers. The cancers found in cartilage, fat, muscle, tendons and bones are called Sarcoma. Examples of Sarcoma are osteosarcoma (bone) and chondrosarcoma (cartilage). Lymphoma is found in the glands of the lymphatic system. It can affect the organs like brain and breast. Leukemia is cancer of bone marrow. Myeloma is found in the plasma cells of bone marrow. In some cases, it forms a single tumor called Plasmacytoma and collects in one bone.

Biomedical Imaging has seen a lot of technological developments in the last few decades [2]. The most vital contributor in early detection of cancer lies with imaging along with the patient's history and physical examination. A single imaging technique cannot achieve cancer detection and may provide ambiguity, resulting in false positives which call for reiteration of imaging techniques. A biomedical imaging technique is competent, if it can verify or eliminate the presence of cancer. Furthermore it is used to examine the growth, plan and evaluate the efficacy of treatment. Biomedical Imaging techniques like Computed Tomography (CT), Magnetic resonance imaging (MRI), Positron-EmissionTomography (PET), Single-Photon Emission -Tomography (SPECT), and Ultrasound (US) are crucial in diagnosis, treatment and prognosis of cancer.

\section{BIOMEDICAL IMAGING TECHNIQUES}

Cancer is been dignosed and treated followed by a number biomedical imaging techniques. This section discusses an overview of biomedical imaging techniques.

\subsection{Computed Tomography (CT)}

Computed Tomography (CT) produces a cross-section ("slices") of anatomy of the human body. Radiographic beams are made incident on the human body. The reflected radio beams create a detailed computerized picture taken with a specialized X-ray machine. CT is more precise than a standard $\mathrm{X}$-ray, and provides a clearer image. Figure 1 shows a CT scan of transverse view of the brain.

The CT system consists of a power-driven table which moves the patient through a circular opening. An X-ray source and a detector assembly within the system rotate around the patient. A single rotation typically takes a second or less. A fan-type Xray passes through the patient's body. Detectors are placed 
opposite the X-ray source absorb the energy and creates an image [3, 4]. Many "snapshots" (at many angles through the patient) are collected during one complete rotation. For each rotation of the X-ray source and detector assembly, the image data are sent to a computer to rebuild all of the individual "snapshots" into one or multiple cross-sectional images (slices) of the internal organs and tissues [5]. Figure 2 shows the CT images for multiple slices of the brain. The minimum and maximum number of CT slices range from 1 to 64 and 320 [5, 6]. CT is one of the most commonly used imaging modality for diagnosis of pulmonary diseases, coronary artery calcification, and metopic craniosynostosis diagnosis of pediatric CT scans of head , Change assessment for spine imaging [7-10]. CT is also used in radiation therapy and planning prostate cancer radiotherapy $[7,11]$. The latest research trends in CT are in areas of registration, segmentation of veins, tumors in brain, lesion in liver, aorta, prostrate, reconstruction of CT images, generation of 3D CT images, superposition of cone-based CT images and generation of synthetic 4D CT images for image guided surgery $[4,12-22]$.

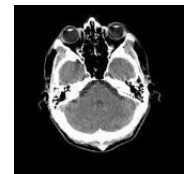

Fig. 1

Transverse views - CT scan of Brain

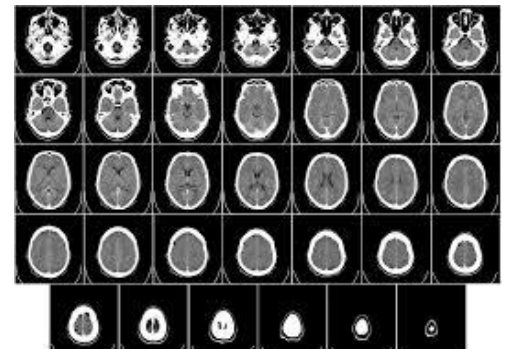

Fig.2 Multiple slices of CT scan of Brain
CT accurately outlines the bone, some soft tissue and blood vessels inside body; it is geometrically accurate, with short scan times and high resolution. CT fails to characterize tissue, transverse slices and there is a restriction on intensity of X-rays for short scan times and lastly the radiation can be harmful and not recommended for patients diagnosed with cancer or pregnant women [12, 23].

\subsection{Magnetic Resonance Imaging (MRI)}

Magnetic Resonance Imaging (MRI) is a noninvasive diagnostic test that takes detailed images of the soft tissues of the body. MRI employs magnetic fields and radio waves to create the images. The human body is made up of $80 \%$ water which contains millions of hydrogen atoms which are magnetic. The magnetic field and radio waves disrupt the orientation and polarity of the hydrogen atoms [24-26]. The time taken by the hydrogen atoms to return to their original alignment is measured by a detector. In brief, MRI measures the water content (or fluid characteristics) of different tissues, which is processed by the computer to create a black and white image. Figure 3 indicates a MRI image for a side view of the brain.

MRI provides in depth image indicating the smallest abnormality. The thickness of slice can be of quarter inch size. A contrast dye which has magnetic properties may be injected into the bloodstream to make the image more distinct. MRI imaging cannot detect blood in arteries and this creates black holes on the image. Angiogram MRI is another form of MRI which can be used to view arteries and veins. Contrast dye may also be used here to image the veins and arteries. Figure 5 shows a MRA scan for the head. MRA can detect aneurysms, blockages of the blood vessels, carotid artery disease, and arteriovenous malformations.
Bone marrow, spinal fluid, blood, liver and soft tissues can be effortlessly imaged with MRI. It is one of the primary diagnostic imaging techniques for detection of brain tumors, spinal cord injury, hypertension, multiple sclerosis, the causes of headache, Crohn's diseases, liver diseases, Alzheimer's disease, dementia [27-34]. Figure 6 displays a MRI image of lumbar spine.

MRI is totally safe as it does not involve any radiation. Some people are sensitive to the contrast dye used and may develop an allergic reaction. MRI scans cannot be used for people with cardiac pacemaker or artificial heart valve metal plate, pin, or other metallic implant piercings (particularly body piercing) intrauterine device, such as Copper-7 IUD, insulin or other drug pump aneurysm clips and during pregnancy. Any metallic substance in the body can affect the quality of the images. Some promising research in MRI Imaging is 3D segmentation, multi-spectral segmentation, MRI guided surgery, MRI guided radiation therapy, Compressed Sensing, MRI/PET hybrid systems and content based image retrieval [35- 37].

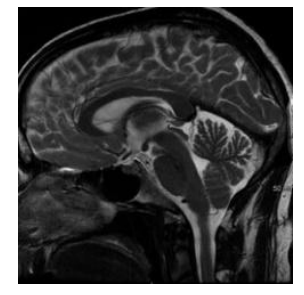

Fig. 3 MRI of the brain
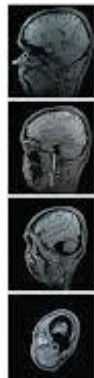
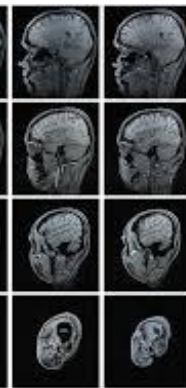

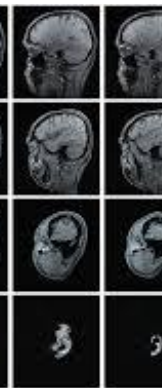

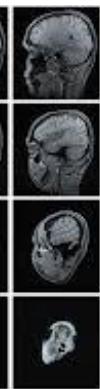

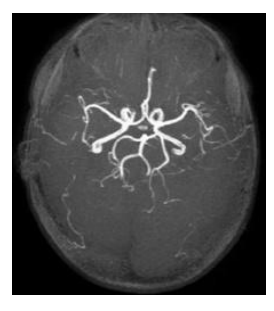

Fig.5 MRA of the brain arteries
Fig. 4 MRI scans of the human brain

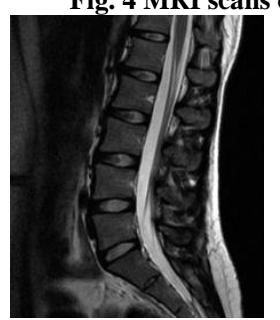

Fig.6 MRI of the lumbar spine

\subsection{Positron Emission Tomography (PET)}

Cancer is a biological processes and molecular imaging cannot be detected with CT or MRI. PET is used to find the progress of cancer cells. The diagnostic accuracy of PET is 8-43\% higher than conventional scans and also affects the treatments planning [38]. A PET scan uses a small amount of radioactive material like FDG (fluorodeoxyglucose) called tracer, which is injected intravenously [39]. The tracer reacts with the cells to produce positrons which cause radiation. With the help of large tunnel shaped scanner, radiation emitted by the positrons is measured and images are generated with the help of a computer. The color variation is seen due to variation in the number of positrons. Figure 7 shows a PET scan of the neck, chest and abdomen region. A PET provides the functional information of organs and tissues. PET scans are very helpful in detection and treatment planning of Epilepsy, Alzheimer's disease, lung and liver cancer and breast cancer and Heart diseases [40-46].

PET imaging is frequently used in both clinical and biological research applications as it can detect cellular changes at a very 
early stage and the radio tracer used is very safe since it does not involve any kind of radiation [47]. However, PET imaging takes a longer time than CT or MRI because the radio tracer has to travel to all parts of the body and the resolution is less and the images are blurring [48].

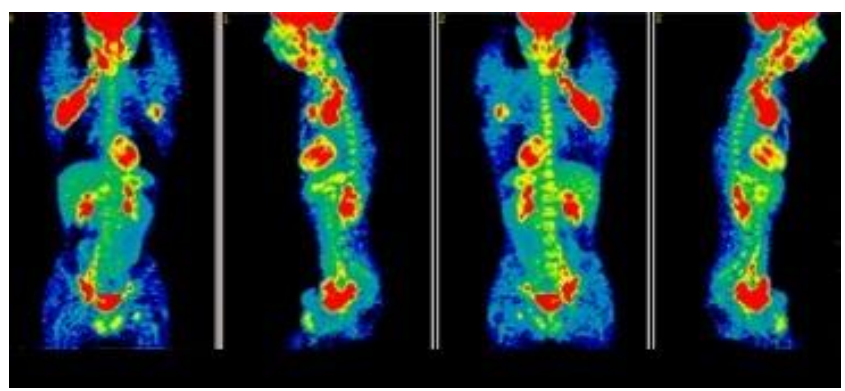

Fig.7 PET scan image

Future work in PET Imaging is generation of high resolution PET images, engineering the next generation PET Detectors, design of hybrid PET scanners, generation of 4D images and design of compact PET scanners [49-54].

\subsection{Single Photon Emission Computed Tomography (SPECT)}

SPECT uses different radioactive tracers and generates three-dimensional image based on its distribution and detection of single photons acquired from multiple planar images. SPECT integrates CT and PET technologies. SPECT provides both functional and anatomical information. For example, brain injuries have reduced or increased blood flow to some parts, which can be easily detected with SPECT. Figure 8 shows some SPECT images of the brain [55-58].

SPECT used to detect seizures, stress, spondylolysis, ischemic areas, thyroid cancer, Alzheimer's disease, Parkinson's disease, Bone imaging and Epilepsy [59-63].

Moreover, the observation time for SPECT lies between several hours to several days. The radioactive tracer used is rightly registered in space and time which gives good sensitivity with spatial and temporal resolution. Sensitivity of SPECT imaging is limited by the scanner design because for every single photon must be detected, hence this calls for precise and accurate design of the scanner. Furthermore, there is cross-talk due to use of different radio tracers and segmentation is challenging [59]. Some of the research challenges involved in SPECT systems are to reduce acquisition time for dynamic imaging, design of compact SPECT scanners, better sensitivity and temporal resolution and compressive sensing [58-65].

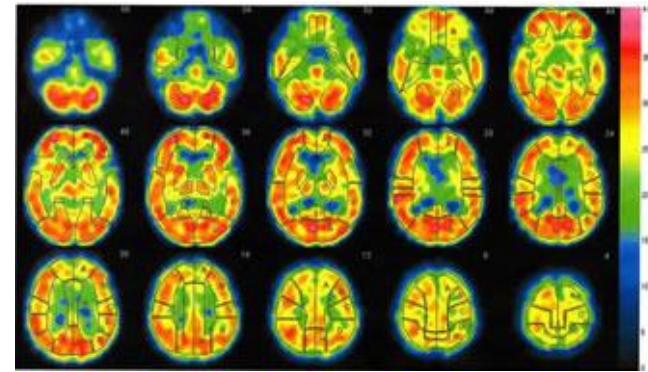

Fig.8 SPECT scan of Brain

\subsection{Functional MRI (FMRI)}

FMRI is a non-invasive imaging technique specifically used to understand the functionality of the brain. Small variation in blood flow is observed as a task is performed by the brain. FMRI detects the difference in the signal due to blood flow. In precise, FMRI detects the brain in action (e.g., speaking or moving) [66-68]. In particular, it indicates which part of the brain is handling critical functions such as thought, speech, vision, movement and sensation [69].

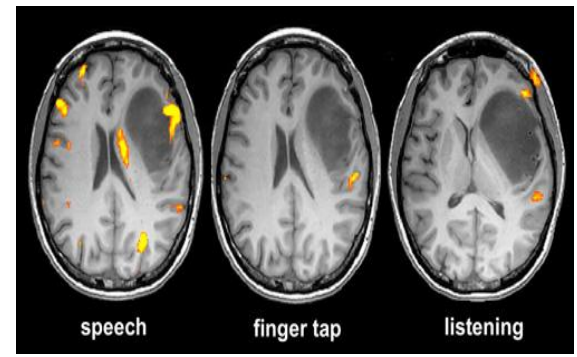

Fig.9 In FMRI, brain areas "light-up" when performing certain tasks

FMRI helps to understand the effect of tumor or stroke on the brain and detection of Alzheimer's disease. Also, helps in planning brain surgery. Figure 9 shows a sample FMRI scan of the brain. The colored areas indicated increased blood intake. Some research advances in FMRI include slice-timing effect and their effect on FMRI, effect of tumor on brain functionality, presurgical planning and extracting semantic data [70-74].

\subsection{Ultrasound (US)}

An ultrasound machine creates images called sonograms by passing high-frequency in the range of $1-20 \mathrm{MHz}$ sound waves through body [75]. Sound waves are reflected back from organs and tissues and transform to images are displayed on a computer [76]. The reflection of sound waves depends on density the tissue, cyst or tumor. Ultrasound can image soft tissues, fluid-filled cysts, tumors which go undetected with Xrays. Ultrasound has some limitations like low sensitivity and limited coverage as cannot pass through bones.

Unlike other blood flow imaging techniques, Color Doppler US is a special non invasive technique that does not use contrast dye, but can produce colored images based unequal blood flow in tumors compared to that in normal tissue. It can indicate the spread of cancer in other parts like liver and pancreas. It is also used to diagnose problems of the liver, gallbladder (such as gallstones), pancreas, thyroid gland, lymph nodes, ovaries, prostrate, kidneys, bladder, breast, abnormal widening of blood vessels (aneurysms), cysts and tumors $[77,78]$.

Ultrasound is a very safe procedure as does not involve any kind of radiation or nuclear elements. It cost much less than CT or MRI. The efficacy of the results depends on expertise of the doctor, which is not the case with CT or MRI. Good images are harder to get in people who are obese. The research challenges in ultrasound imaging is generation of 3-D images to accurately differentiate benign and malignant tumors and ultrafast ultrasound imaging (4D imaging) for moving organs like heart and liver [79-82]. Lastly use of ultrasound images in surgery and planning of cancer and molecular targeting is newest development in Ultrasound research [83, 84]. 


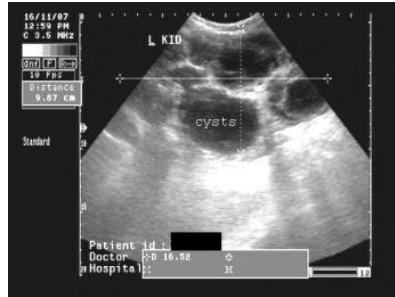

Fig.10 Ultrasound scans showing cysts in kidney

\section{MEDICAL IMAGE FUSION}

Medical image fusion is the process of combining two or more image modalities into a single image. The resulting image is more informative than the individual images. The quality of the resultant image is improved due to reduced uncertainty and redundancy. There is need of skilled personnel for interpretation of biomedical images. There are chances of incorrect interpretations from the images due to its inherent limitations. Moreover, a number of diagnostic tests may be done to make certain of medical problem. There is always a demand on the time for accurate diagnosis and treatment. This can be achieved by Medical Image fusion. Image Fusion provides comprehensive morphological and functional information which reflects physiological and pathological changes. The images which can be fused may have the same information from different views or images with complementary information can be considered for image fusion. With Image fusion, the clinical applicability for diagnosis and treatment of medical problem can be effortlessly developed. With advances in image processing, medical image fusion is an emerging stream of research in biomedical applications $[2,85]$.

Image Fusion can be performed on images acquired from different sources, at different times, with different resolutions and with different focus [86-90]. The images can be fused in 3 ways: pixel-level based image fusion, feature-level based image fusion and decision-making. In pixel level fusion, the input images are fused pixel by pixel followed by the information extraction. In feature level fusion, the certain features are extracted from each input image separately and then fused. Decision level fusion combines the results from multiple algorithms to yield a final fused decision [91-95].

Doctors make the most by analyzing the spatial and physiological relationships between the modalities, but this requires expertise. The incapacity to visually understand the different imaging modality separately is the main reason for evolution of biomedical image fusion. The images may contain different information like different color or shape. The same entity may appear different in different images. The fusion of these imaging modalities to one image is the ideal to interpret them well. Presently, Doctors still analyze images discretely. Image Fusion gives an amalgamated image which indicates all the details in one image. By means of biomedical image fusion, all images are stored in digital form and this helps in easy management of patient's database for the hospitals. This helps the doctors in earlier diagnosis and treatment planning. The cost of CT, MRI and ultrasound scanners is high and since they work on different concepts, it is complex to integrate all the three imaging techniques in one machine. Also, not many hospitals have all these facilities at one place. This is a good reason for considering biomedical image fusion, which will be a software technique and not much hardware is involved.
Image fusion will also play a major role in planning radiation therapy and radiosurgery [96]. Despite the many advantages of image fusion is not being used as yet in India. Limiting factors include: (i) cost intensive investment in hardware and software, (ii) time-consuming fusion procedures (10 to $45 \mathrm{~min}$.), (iii) new $3 \mathrm{D}$ visualization techniques are needed to communicate the results to the clinicians, (iv) image registration and fusion tools are still evolving and are more computation intensive, and (v) CT, MRI, and PET data sets must be easily be transferred to one common imaging workstation. Finally, the objective and quantitative evaluation of spatial errors is an important task for image fusion is to gain clinical impact [104].

\section{APPLICATIONS OF IMAGE FUSION}

Medical Image Fusion has been used in detection, diagnosis and treatment planning of brain tumor and breast cancer. The following section discusses the various imaging techniques and to evaluate the performance of different image fusion.

\subsection{Brain Tumor}

Brain Tumor is a group of abnormal cells found in the brain cells, membranes around the brain, nerves and glands of the brain. Tumors can directly destroy brain cells, producing inflammation, placing pressure on other parts of the brain and increasing the pressure on the skull. CT, MRI, PET and SPECT are some of the commonly used imaging techniques. CT has been the keystone in detection of brain tumor in biomedical imaging. It is the easiest imaging technique with highest resolution but fails to show the essential differences in physiology. MRI is performed to study the damage caused to the soft tissue of the brain and fails to provide information about bones or skull. The fusion of these two imaging techniques will be of great help for radiotherapy planning as planning is done on CT images and localization is better with MRI images. The fused image improves diagnostic accuracy and localization of tumors all available in a single image [105107].

The metabolic activity of the tumor cells can be studied by PET imaging. However, it fails to provide higher-resolution and anatomic information. For many years, separate procedures were carried for CT and PET scans. An expert would provide his views based on his mental synthesis of the images. There are multitude problems with these imaging techniques such as different positioning of the patient and different procedures. More recently, computers perform the fusion of these images. Nowadays, with the state-of-art scanners, CT-PET scans are performed in one machine and hence it is easier to get the images which are in the same form with minimal misregistration. Early detection of brain tumors is possible on MRI and CT but tumor grading remains a difficult task [98]. FDG- PET can differentiate between high-grade (hyper metabolic) and low-grade (hypometabolic) tumors with high accuracy; however, it does not distinguish between low-grade tumors and some benign lesions. Another multi-modality image fusion technique is PET-MRI, which can be used to define biopsy targets and separate the tumor cells from the affected cells. This also helps to determine the residual tumors and predict recurrence of tumors [103]. In radiation therapy of brain tumor, SPECT is used to differentiate the functional areas and non-functional areas of the brain. SPECT ensures to avoid high dosages of radiotracer element to functional parts of the brain [86]

Tan Haibo, Chen Limin, Guan Yihui and Lin Xiangtong et al compared MRI, PET and PET/CT imaging techniques for 55 patients with suspected brain tumor. The sensitivities of were $87.2 \%, 76.2 \%$ and $92.3 \%$ respectively and their specificities 
were $81.3 \%, 62.5 \%$ and $87.5 \%$ respectively for the 3 imaging techniques [99,-101]. Michael A Fisher et al evaluated the diagnostic accuracy of whole body MRI and PET/CT imaging techniques. Sixty-eight patients underwent PET/CT for staging of malignancy. PET/CT revealed 48/64 (75\%) cases of cancer. The Detection rates and positive predictive value of MRI alone was $64 \%$ and $84 \%$ and for PET/CT it was $57 \%$ and $93 \%$. Detection rates and PPV for side-by side analysis without and with fused images were $72 \%$ and $89 \%$ and $74 \%$ and $91 \%$ respectively [102].

Christian Buchbender, Till A Heusner et al have compared multi imaging modalities like PET/MRI and PET/CT. Cerebral metastases represent the most frequent brain tumor which occur in $20 \%-40 \%$ of cancer patients. PET alone cannot compensate the shortcomings of CT or MRI. PET could detect only $61 \%$ of metastases compared to that were detected by MRI. PET/CT was found weak in terms of maximum sensitivity, specificity and accuracy of $50 \%, 97 \%$ and $76 \%$ when MRI was used as a benchmark [103]. Figure 12-14 indicates CT, MRI, CT/ MRI fused, MRI, PET and MRI/PET fused images [104].
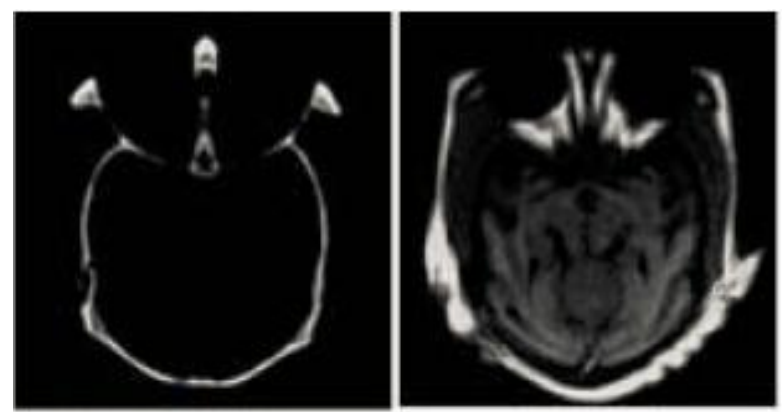

Fig.11 CT and MRI Image

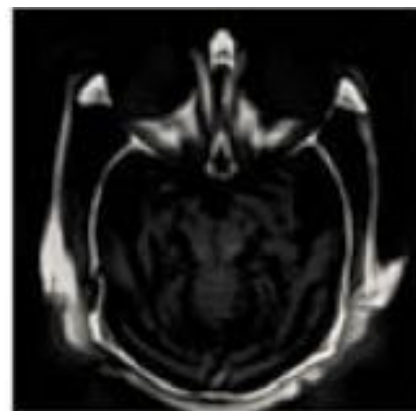

Fig.12 Fused CT \& MRI Images
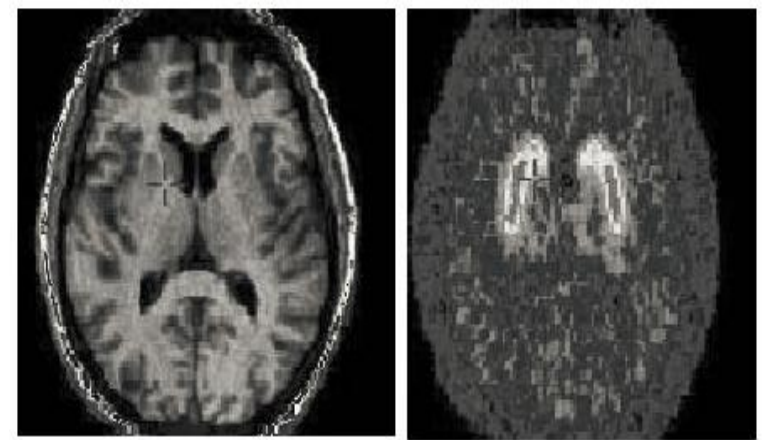

Fig.13 MRI and PET Images

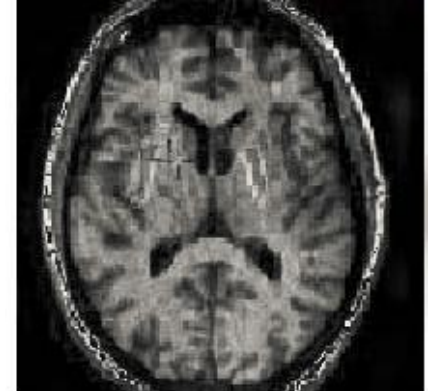

Fig.14 Fused MRI/PET Image

\subsection{Breast Cancer}

Mammography, Breast Ultrasound, Digital tomosynthesis mammography, Breast Thermography, MRI and PET are some of commonly used imaging techniques for detection of Breast Cancer. Mammography is similar to X-rays, but uses low doses amplitude X-rays. Cancerous cells appear brighter o the mammogram. A modification of mammography which generates $3 \mathrm{D}$ view of the breast is called Digital tomosynthesis mammography. In cases, cancers go undetected with Mammography. Breast Ultrasound is used in case dense breast tissue. The drawback with Ultrasound Imaging is that the resolution is less. For grading of breast cancer, PET is widely used imaging technique.

Linda Moy et al evaluated the fusion of MRI and FDG-PET images for detection of breast cancer. They considered 36 women (i.e. of age range of $24-65 y e a r s$ and mean age 43years) with 90 lesions detected on MRI. They found the following results - Sensitivity of MRI alone ne was 95\%, FDGPET alone was $57 \%$ and fusion was $83 \%$. The specificity increased from $57 \%$ to $97 \%$. The false-negative rate on FDGPET alone was $26.7 \%$ and after fusion was reduced to $9 \%$. The positive predictive value (PPV) in MRI was $77 \%$, increased to $98 \%$ with fusion [105].

PET-CT is another multi modality imaging technique used in detection of Breast Cancer. Isabelle Segaert, Felix Mottaghy et al have evaluated the accuracy of PET-CT against PET and CT individually in conventional staging in breast cancer. Sensitivity of PET/CT was $97.1 \%$ against $87.5 \%$ in case of PET. The nodal status was increased to $87.5 \%$ from $62.5 \%$. Specificity and negative predictive value for nodal status were $100 \%$ and $66.6 \%$, respectively. PET/CT is able to visualize most clinical stage IIB and III primary breast cancers. PET/CT is superior to conventional staging for detecting internal mammary chain nodes and metastatic disease, but not for axillary staging. [106]. T A Heusner et al studied the accuracy of PET MRI mammography (MRM) for 58 breast lesions. The sensitivity, specificity, PPV, NPV and accuracy were 93\%, $60 \%, 87 \%, 75 \%$ and $85 \%$ for MRM, respectively. For FDGPET/MRM they were $88 \%, 73 \%, 90 \%, 69 \%$ and $92 \%$, respectively. Figure 15-17 indicates MRI, PET and fused MRI/PET images [107,108]. 


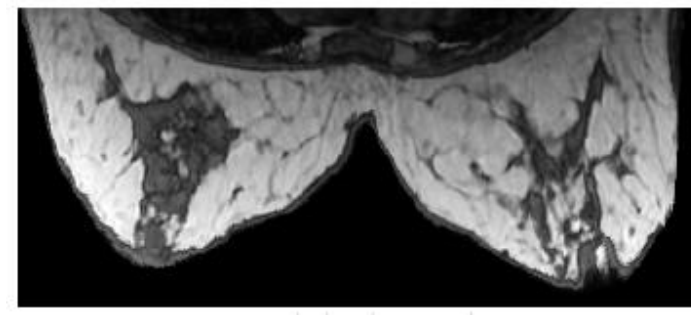

Fig. 15 MRI Image of Breast

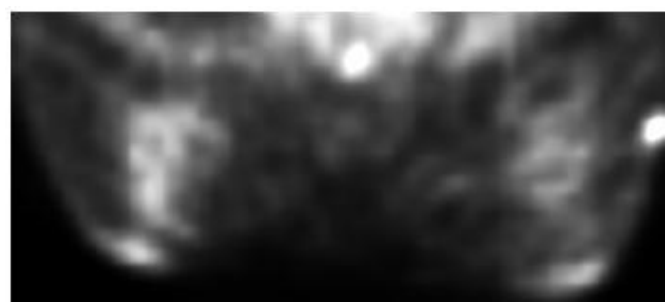

Fig. 16 PET image of breast

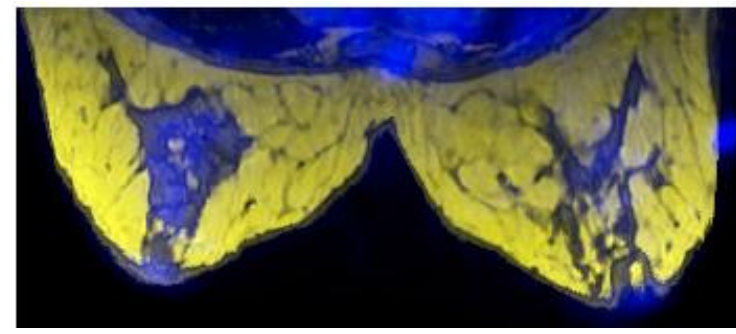

Fig. 17 MRI/PET Fused image with color mixing

\section{CONCLUSION}

The commonly used biomedical imaging techniques for diagnosis and treatment of Cancer were discussed in detail along with their applications, advantages, disadvantages and research challenges. The need for biomedical image fusion is discussed and the various modalities used for image fusion was discussed. It is seen that results of fused images are better than individual imaging techniques.

\section{ACKNOWLEDGEMENT}

I would like to acknowledge Dr.Rajesh, Specialist in Radiology, for his valuable inputs in the domain of Biomedical Imaging. I would also like to thank Dr.Jaydatt, Oncology India to provide inputs in diagnosis of Cancer and some real time benefits Image Fusion would provide in Cancer management.

\section{REFERENCES}

[1] http://mattersindia.com/1-million-indians-get-cancerannually/

[2] Leonard Fass, "Imaging and cancer: A review," Molecular Oncology 2 (2008) $115-152$.

[3] David J.Brenner and Eric J. Hall, "Computed Tomography - An increasing source of Radiation Exposure", The New England Journal of Medicine, 2007.

[4] Jiang Hsieh, Brian Nett, Zhou Yu, Ken Sauer,JeanBaptiste Thibault, Charles A. Bouman," Recent Advances in CT Image Reconstruction," Advances in CT Imaging, Springer Science 2013. [8]

[5] "Multi-Slice CT Scanners," Technology Update No.1, 2nd Edition, Jan 2002. [19]
[6] Thomas Flohr and Bernd Ohnesorge, "Multi Slice CT Technology ", Book Chapter.

[7] Jun Lai, Qingjie Wei, "Automatic lung fields segmentation in CT scans using morphological operation and anatomical information," Bio-Medical Materials and Engineering 24 (2014) 335-340.

[8] Yiting Xie, Matthew D Cham, Claudia Henschke, David Yankeleviz, Anthony P Reeves, " Automated coronary artery calcification detection on low-dose chest CT images," Proc.SPIE9035, Medical Imaging 2014.

[9] Hua Li et al, "Automatic CT simulation optimization for radiation therapy: A general strategy," The International Journal of Medical Physics Research and Practice, 41, 031913 (2014).

[10] Carlos S Mendoza et al, "An optimal set of landmarks for metopic craniosynostosis diagnosis from shape analysis of pediatric CT scans of the head," Proc. SPIC8670, Medical Imaging 2013.

[11] Lorenz C et al, "Change assessment for CT Spine Imaging," Biomedical Imaging (ISBI), 2013 IEEE $10^{\text {th }}$ International Symposium on Biomedical Imaging from Nano to Macro, April 2013.

[12] Oscar Acosta, Jason Dowling, Giael Drean, Antoine Simon, Renaud de Crevoisier and Pascal Haigron, " MultiAtlas-Based segmentation of Pelvic Structures from CT Scans for Planning in Prostrate Cancer Radiotherapy," Abdomen and Thoracic Imaging: An Engineering \& Clinical Perspective, 2014.

[13] Andrew D. Perron, "How to read a Head CT Scan," Book Chapter. Ch069-X2872.Indd, 2008.

[14] Pavel Dvorák, Walter G. Kropatsch, Karel Bartusek, "Pathological Area Detection in MR Images of Brain," Elektrorevue, Vol.4, No.1, April 2013.

[15] Xiachuan $\mathrm{He}$ et al, "Veins Segmentation and ThreeDimensional Reconstruction from CT Images Using Multilevel OTSU Method," Image and Graphics (ICIG), 2013 seventh International Conference on Image and Graphics, July 2013.

[16] Mingna Zheng, J. Jeffery Carr, Yaorong Ge, " Automatic Aorta Detection in Non-contrast 3D Cardiac CT Images Using Bayesian Tracking Method," Medical Computer Vision, Large Data in Medical Imaging, 2014.

[17] Yinghuan Shi et al, "Prostrate Segmentation in CT Images via Spatial-Constrained Transductive Lasso," (CVPR), 2013 IEEE International Conference on Computer Vision and Pattern Recognition

[18] Jan Rosell and Paolo Cabras, "A three-stage method for the $3 \mathrm{~d}$ reconstruction of the tracheobronchial tree from CT Scans," Computerized Medical Imaging and Graphics, Vol. 37, Issue 7, Oct 2013.

[19] MGP Cavalcanti, SS Rocha and MW Vannier, " Craniofacial measurements based on 3D-CT volume rendering: implications for clinical applications," A Journal of Head \& Neck Imaging, 2014.

[20] Landis K. Griffeth, "Use of PET/CT scanning in cancer patients: technical and practical considerations," BUMC Proceedings 2005; 18:321-330. 
[21] LHS Cevidanes et al, "Superposition of 3D cone-beam based CT models of orthognathic surgery patients," A Journal of Head \& Neck Imaging, Volume 34 Issue 6, Nov 2005. [14]

[22] Feng P.Li et al, "Generation of synthetic 4D Cardiac CT Images for Guidance of Minimally Invasive Beating Heart Interventions," Information Processing in Computer Assisted Interventions, Volume 7915, 2013. [15]

[23] Benjamin P. Jonker, "Image fusion pitfalls for cranial radiosurgery," Surgical Neurology International, Open Access, 17 April, 2013.[2]

[24] Panagiotis Vartholomeos and Constantinos Mavroidis, "Simulation Platform for Self-Assembly Structures in MRI-guided Nanorobotic Drug Delivery Systems," 2010 IEEE International Conference on Robotics and Automation Anchorage Convention District, May 3-8, 2010.

[25] Michael C. Steckner," Advances in MRI Equipment Design, Software and Imaging Procedures", 2006.

[26] Tal Geva, "Magnetic Resonance Imaging: Historical Perspective," Journal of Cardiovascular Magnetic Resonance (2006) 8, 573-580.

[27] Hassan Khotanlou et al, "3D brain tumor segmentation in MRI using fuzzy classification, symmetry analysis and spatially constrained deformable models," Fuzzy sets and systems 160 (2009) 1457-1473.

[28] Xavier Llado, Aranu Oiver, " Segmentation of multiple sclerosis lesions in brain MRI: A Review of automated approaches", Information Sciences, Vol. 186, Issue 1, March 2012

[29] Nan Zhang, Su Ruan, Stephane Lebonvallet, "Kernal Feature selection to fuse multi-spectral MRI Images for brain tumor segmentation," Computer vision and Image Understanding, Volume 115, Issue 2, Feb 2011, Pages 256-269.

[30] Wolf-Dieter Heiss, Peter Raab and Heinrich Lanfermann, "Multimodality Assessment of Brain Tumors and Tumor Recurrence, "The Journal of Nuclear Medicine, August 12, 2011.

[31] Anthony Bozzo, Judith Marcoux, Mohan Radhakrishna, "The role of Magnetic Resonance Imaging in the management of Acute Spinal Cord Injury", Journal of Neurotrauma, Aug 2011.

[32] Amy E Burchell, Laura E Ratcliffe et al, "Utility of MRI as the primary imaging tool in hypertension," Journal of Cardiovascular Magnetic Resonance 2014.

[33] Mahapatra D, Schuffler P J, Tiebeek," Automatic Detection and segmentation of Crohn's disease Tissues from abdominal MRI," Medical Imaging, IEEE Transactions on Medical Imaging, Vol. 32, Issue 12, Dec 2013.

[34] Saraswathi S, Mahanand B S, "Detection of onset of Azhemier's disease from MRI images using a GA-ELMPSO classifer," 2013 IEEE Computational Intelligence in Medical Imaging, 2013 IEEE forth International Conference.

[35] Haldar J P, Hernando D, Zhi-PE Liang, "CompressedSensing MRI with Random Encoding," IEEE Transactions on Medical Imaging, Volume 30, Issue 4, April 2011.
[36] Habib Zaid and Alberto Del Guerra, "An outlook on future design of hybrid PET/MRI systems", Medical Physics 38, 5667(2011).

[37] Abraham Varghese, Kannan Balakrishnan, Reji R Verghese and Joseph S Paul," Content Based Image Retrieval of T2 Weighted Brain MR Images similar to T1 Weighted Images," Pattern Recognition and Machine Intelligence, Volume 8251, 2013. Journal of Innovative Research in Electrical, Electronics, Instrumentation and Control Engineering, Vol.2, Issue 1, January 2014.

[38] P.Moskal, P.Salabura, M.Silarski, et al, "Novel detector systems for Positron Emission Tomography," Proceedings of MCSB 2010 Conference, Krakow, 21-22.05.2010.

[39] Michael E Phelps, "Positron emission tomography provides molecular imaging of biological processes," Proceedings of the National Academy of Science of the United States, 2000.

[40] Mian M Aladdin, "Positron Emission tomography (PET) imaging with F-based radiotracers," American Journal of Nuclear Medical Imaging, 2011.

[41] Wallach D, Lamare F, Kontaxakis G, Visvikis,"Super resolution in Respiratory Synchronized Positron Emission Tomography," IEEE Transactions on Medical Imaging, Vol.30, Issue 2, Feb 2012.

[42] Jaewon Yang, Tokihiro Yamamoto, Samuel R Mazin, et al,"The potential of PET for intra treatment dynamic lung tumor tracking: A phantom study," The International Journal of Medical Physics Research and Practice, Vol 41, Issue 2, 2014. [48]

[43] Atsushi Teramoto, Hiroshi Fujita et al, "Hybrid method for detection of pulmonary nodules using PET/CT a prelimary study," International Journal of Computer R Radiology and Surgery, Vol. 9, Issue 1,pp 59-69, Jan 2014.

[44] Lartizien C, MArache Francisco, Prost, "Automatic detection of Lung and Liver Lesions in 3D PET Images: A Pilot Study," IEEE Transactions on Nuclear Science, Vol. 59,Issue 1, 2012

[45] Jianhua Yan, Chaal J, Majewski,Vaigneur K, "Initial study design of a breast-dedicated PET scanner with biospy capability using GATE," Nuclear Science Symposium and Medical Imaging Conference, 2011 IEEE.

[46] Marlene Rossibel et al, "Assessment of inflammation in large arteries with 18F-FDG-in elderly," Computerized Medical Imaging and Graphics, Vol.37, Issues 7-8, Oct 2013.

[47] Jan Bucerius et al," Prevalence and Risk Factors of Carotid Vessel Wall Inflammation in Coronary Artery Disease Patients," JACC Journals, Vol.4, Issue 11, Nov. 2011.

[48] Ciprian Catana, Daniel Procissi, Yibao Wu et al, " Simultaneous in vivo positron emission tomography and magnetic resonance imaging, " Proceedings of the National Academy of Science of the United States, 2007.

[49] Chih-Yu Hsu, Lai, Yeong-Lin, Chih-Cheng Chen, Yu-Tzu Lee, "Image Segmentation Method with PET Time Sequence Images", 2011 Second International Conference 
on Innovations in Bio-inspired Computing and Applications.

[50] Giampaolo Tomasi, Federico Turkheimer, Eric Aboagye," Importance of Quantification for analysis of PET data in Oncology: A Review of Current methods and trends in future", Journal of Molecular Imaging and Biology, 2011.

[51] Alexender V. Stolin, Stan Majewski, Gangadhar Jaliparthi and Raymond R Raylam," Construction and Evaluation of a ;Prototype High Resolution, Silicon PhotomultiplierBased, Tandem Positron Emission Tomography System," IEEE Transactions on Nuclear Science, Vol.60, No.1, Feb 2013.

[52] N.Jon Shah, Hans Herzog, Christoph Weirich et al, "Effects of Magnetic Fields of up to 9.4T on Resolution and Contrast of PET Images as Measured with an MRBrainPET," PLOS ONE,Vol.9, Issue 4, April 2014.

[53] Breuilly M, Malandain G, Ayache N, Guglielmi J, "Image based motion detection in $4 \mathrm{D}$ images and application to respiratory motion suppression" 2013 IEEE 10th International Symposium on Biomedical Imaging.

[54] Arne Vandenbrocke and Craig S. Levin, "Engineering the Next-Generation PET Detectors," Book Chapter, pp 761798, 2014.

[55] Studenski M.T, Gilland D R, Cebula, "Acquisition and Processing Methods for Bedside Cardiac SPECT Imaging System," IEEE Transactions on Nuclear Science, Vol.57, Issue 1,Feb 2010.

[56] Arman Rahmim and Habib Zaidi, "PET versus SPECT: strengths, limitations and challenges", Review article, Molecular Medicine Communications, 2008.

[57] Peeyush Bhargava, Guocheng He, Amin Samarghandi, Ebrahim S Delpassand, " Pictorial review of SPECT/CT imaging applications in clinical nuclear medicine" American Journal of Molecular Imaging 2012, 2(2):221231.

[58] Mark T Madsen, "Recent Advances in SPECT Imaging," Journal of Nuclear Medicine, Jan 2007.

[59] Toshiyuki Aoi, Tsutomu Zeniya, Hiroshi Watabe, Hossain M Deloar, Tetsuya Matsuda and Hidehiro Iida, "System design and development of a pinhole SPECT system for quantitative functional imaging of small animals," Annals of Nuclear Medicine Vol. 20, No.3, 245-251,2006.

[60] Piotr J Slomka, James A Patton, Daniel S Berman and Guido Germano, "Advances in technical aspects of myocardial perfusion SPECT imaging," Journal of Nuclear Cardiology, March/April 2009.

[61] Baodong Liu, Akiva Mintz and Hengyong Yu, "Real Phantom Datasets for the Evaluation of Compressive Sensing based interior SPECT," Journal of Bio Medical Imaging, Sept 2004.

[62] Tara Barwick, Iain Murray, Hakim Megadmi et al, "Single photon emission computed tomography/Computed tomography using Iodine-123 in patients with differentiated thyroid cancer: additional value over whole body planar imaging and SPECT," European Journal of Endocrinology, 1131-1139, 2011

[63] C la Fougere, A Rominger, S Forster, J Geisler, P Bartenstein, "PET and SPECT in epilepsy: A critical review," Journal on Epilepsy and Behavior 15(2009) 5055 .

[64] H Zaidi, "Organ Volume Estimation Using SPECT," IEEE Transactions on Nuclear Science, Vol.43, No.3, June 1996.

[65] Baodong Liu, Akiva Mintz and Hengyong Yu, "Real Phantom Datasets for the Evaluation of Compressive Sensing based interior SPECT," Journal of Bio Medical Imaging, Sept 2004.

[66] Martin A Lindquist, "The Statistical Analysis of fMRI Data," Statistical Science, 2008, Vol.23, No.4, 439-464.

[67] Peter Jezzard and Ahmed Tossy, "Functional MRI," Book Chapter.

[68] Dan Lloyd, "Functional MRI and the study of Human Consciousness," Journal of Cognitive Neuroscience, 2002

[69] Ronald Sladky, Karl J Friston, Jasmin Trostl, Ross Cunnington, Ewald Moser, Christian Windischberger, "Slice-timing effect and their correction in functional MRI," Journal of NeuroImage 58(2011) 588-594.

[70] Michael D Greicius, Gaurav Srivastava, Allan L Reiss, "Default-mode network activity distinguishes Alzheimer's disease from healthy aging: Evidence from fMRI," PNAS, vol.10, no.13, March 2004

[71] Smits M, "Functional Magnetic Resonance Imaging in Brain Tumor," NeuroOncology Magazine 2012 2(3) 123128.

[72] Francisco Pereira, Matthew Botvinick, "A systematic approach to extracting semantic information from fMRI data," NIPS Conference, 2012

[73] Weier Li, Scott D Watt, Robert J OGG, et al "Functional magnetic resonance imaging of visual cortex performed in children under sedation to assist in presurgical planning," Journal of Neurosurgery, Pediatrics, 2013.

[74] Nicolas Wiest-Daessle, Olivier Commowick, Aymeric Stamm, et al,"Comparison of 3 diffusion models to track the hand motor fibers within the corticospinal tract using functional, anatomical and diffusion MRI," MICCAI 2011 Workshop on Computational Diffusion MRI.

[75] Vincent Chan and Anahi Perlas, "Basics of Ultrasound Imaging," Book Chapter, Atlas of Ultrasound-Guided Procedures in Interventional Pain Management, Springer 2011.

[76] Peter N T Wells and Hai-Dong Llang, "Medical ultrasound: imaging of soft tissue strain and elasticity," Journal of Royal Society Interface 2011

[77] "Ovarian cancer: role of ultrasound in preoperative diagnosis and population screening" Ultrasound Obstetrics and Gynaecology 2012.

[78] Martijn Smeenge, Massimo Mischi, MPilar Laguna Pes,Jean J M, Hessel Wijkstra, "Novel Contrast-enhanced ultrasound imaging in prostate cancer", World journal on Urology (2011) 29,581-587.

[79] A P Ayyappan, S Kulkarni, P Crystal," Pregnancyassociated breast cancer: spectrum of imaging appearences,"The British Journal of Radiology, 83 (2010), 529-534. and MRI. 
[80] Eddie Yin-Kwee Ng," Breast imaging: A survey," World Journal of Clinical Oncology, April 2011.

[81] Brian C Porter, Deborah J Rubens, John G Strang, " Three-Dimensional Registration and Fusion of Ultrasound and MRI Using Major Vessels as Fiducial Markers," IEEE Transactions on Medical Imaging, Vol. 20, No.4, April 2001

[82] Jeremy Bercoff, "Ultrafast Ultrasound Imaging," Book chapter, Ultrasound Imaging - Medical Applications.

[83] Stamatia Destounis, Mary Newell, Renee Pinsky," Breast Imaging in Over Weight and Obese Patient," AJR Women's Imaging, Clinical Perspective, Oct 2010.

[84] Siver A Moestue, Ingrid S Gribbestad and Rune Hansen, "Intravascular Targets for Molecular Contrast-Enhanced Ultrasound Imaging," International Journal of Molecular Science 2012.

[85] A.P. James, B.V. Dasarathy, " Medical Image Fusion: A survey of the state of art," Information Fusion, 2014.

[86] Roger Lundqvist, "Atlas-Based Fusion of Medical Brain Images, Methods and Applications," Dissertation, Uppsala, University, 2001.

[87] Constantinnos S. Pattichis, Marios S. Pattichis, Evangelia Micheli- Tzanakou, "Medical imaging fusion applications: An overview," 0-7803-7147-X/01 2001 IEEE.

[88] Les R Folio et al, "Automated Registration, Segmentation and Measurement of Metastatic Melanoma Tumors in Serial CT Scans," Academic Radiology, Volume 20, Issue 5, May 2013.

[89] "Data Fusion Techniques - Image Fusion and Algorithm Fusion," Airborne Underwater Geophysical Signals.

[90] Thorsten Twellmann, Axel Saalbach, Olaf Gerstung, Martin O Leach and Tim W Nattkemper, "Image Fusion for dynamic contrast enhanced magnetic resonance imaging," Biomedical Engineering Online, Oct 2004.

[91] Sehkar A S, Giri Prasad M N ,"A novel approach of image fusion on MR and CT images using wavelet transforms," 3rd International Conference on Electronics Computer Technology 2011.

[92] Yihua Lan, Haozheng Ren, Yong Zhang, "Multi-band Vector Wavelet Transformation based Multi-Focus Image Fusion Algorithm," Journal of Software, Vol.8, No.1, Jan 2014.

[93] Ayush Dogra and Manjeet Singh Patterh, "CT and MRI Brain Images Registration for Clinical Applications", Journal Cancer Science \& Therapy, 2014.

[94] B.K Shreyamsha Kumar, "Multifocus and multispectral image fusion on pixel significance using discrete cosine harmonic wavelet transform," SIViP (2013) 7:1125 1143

[95] Firouz Abdullah Al-Wassai, N V Kalyankar, Ali A AlZaky, "Multisensor Image Fusion Based on FeatureLevel," International Journal of Latest Technology in Engineering, Management and Applied Science, vol. 1, no. 5, pp. 124-138, 2012.
[96] Arnold C Paulino, Wade L Thorstad and Timothy Fox, "Role of Fusion in Radiotherapy Treatment Planning," Seminars in Nuclear Medicine, Vol XXXIII, No.3 (July), 2003,pp 238-243.

[97] Andreas H Jacobs, Lutz W Kracht, Axel Gossmann, Maria A Ruger, Anne V Thomas, Alexander Thiel and Karl Herholz, "Imaging in Neurooncology," The Journal of the American Society for Experimental NeuroTherapeutics, Vol 2, 333-347, April 2005.

[98] Suresh Padala, "Fusion of CT and MRI Scanned Medical Images Using Image Processing, "AKGEC International Journal of Technology, Vol 3, No.2.

[99] Tan Haibo, Chen Limin, Guan Yihui and Lin Xiangtong,"Comparison of MRI, F-18 FDG and 11CCholine PET/CT for Their Potentials in Differentiating Brain Tumor Recurrence from Brain Tumor Necrosis Following Radiotherapy", Clinical Nuclear Medicine, Vol 36, Issue 11-pp 978-981,Nov 2011

[100] Arne-Jo"rn Lemke et al, "Retrospective Digital Image of Multidetector CT and F-FDG PET: Clinical value in Pancreatic Lesions - A Prospective study with 104 patients", The Journal of Nuclear Medicine, 2004.

[101] P.Ambika Priyadarshini, M.R Mahalakshmi, "Multimodal Medical Image Fusion Based on SVD", IOSR Journal of Computer Engineering, Jan 2014.

[102] Michael Fisher, Daniel Nanz et al "Diagnostic accuracy of whole-body MRI/DWI image fusion for detection of malignant tumors: a comparison with PET/CT" European Radiology (2011).

[103] Christian Buchbender, Till A Heusner, Thomas C Lauenstein et al, "Oncologic PET/MRI, Part 1:Tumors of the Brain, Head and Neck, Chest, Abdomen and Pelvis,"The Journal of Nuclear Medicine,Vol.53, no.6 928-938, Jun 2012.

[104] M.Malini Deepika, Dr. V Vaithyanathan, "An efficient method to improve the spatial property of medical images," Journal of Theortical and Applied Information Technology, Vol.35, No.2, Jan 2012.

[105] Linda Moy, Marilyn E Noz, Gerald Q Maguire, Amy Melsaether, Abby E Deans, Antoninette D MurphyWalcott BS and Fabio Ponzo, " Role of Fusion of Prone FDG-PET and Magnetic Resonance Imaging of the Breasts in the Evaluation of Breast Cancer, " The Breast Journal, Volume 16, Issue 4, pages 369-376, July/Aug 2010

[106] Issabelle Segaert, Felix Mottaghy, Sarah Ceyssens, Walter De Wever, Sigrid Stroobants et al, "Additional Value of PET-CT in Staging of Clinical Stage IIB and III Breast Cancer," The Breast Journal, Volume 16, Issue 4 , pages 617-624, Nov/Dec 2010.

[107] A Heusner, S Hahn, C Jonkmanns, S Kuemmel, et al, " Diagnostic accuracy of fused PET and MRI mammography: initial results," The British Journal of Radiology, 84 (2011),126-135.

[108] Karl G Baum et al, "Techniques for Fusion of Multimodal Images: Application to Breast Imaging," IEEE ICIP 2006. 